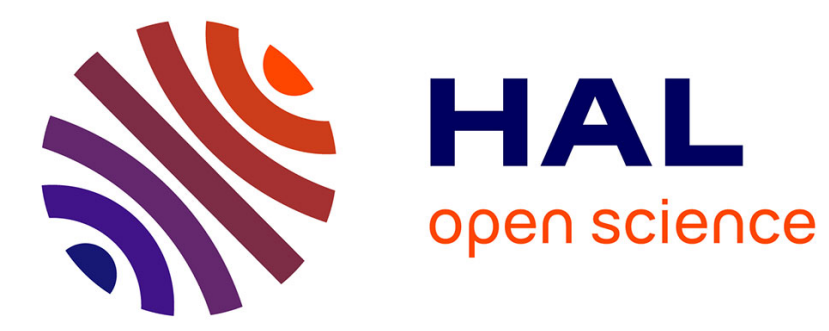

\title{
A non-local void dynamics modeling and simulation using the Proper Generalized Decomposition
}

Chady Ghnatios, Pavel Simacek, Francisco Chinesta, Suresh Advani

\section{To cite this version:}

Chady Ghnatios, Pavel Simacek, Francisco Chinesta, Suresh Advani. A non-local void dynamics modeling and simulation using the Proper Generalized Decomposition. International Journal of Material Forming, 2020, 13 (4), pp.533-546. 10.1007/s12289-019-01490-7 . hal-02974825

\section{HAL Id: hal-02974825 \\ https://hal.science/hal-02974825}

Submitted on 22 Oct 2020

HAL is a multi-disciplinary open access archive for the deposit and dissemination of scientific research documents, whether they are published or not. The documents may come from teaching and research institutions in France or abroad, or from public or private research centers.
L'archive ouverte pluridisciplinaire HAL, est destinée au dépôt et à la diffusion de documents scientifiques de niveau recherche, publiés ou non, émanant des établissements d'enseignement et de recherche français ou étrangers, des laboratoires publics ou privés. 


\title{
A non-local void dynamics modeling and simulation using the Proper Generalized Decomposition
}

\author{
Chady Ghnatios $^{1}$ (D) $\cdot$ Pavel Simacek $^{2} \cdot$ Francisco Chinesta $^{3} \cdot$ Suresh Advani ${ }^{2}$
}

\begin{abstract}
In this work we develop a void filling and void motion dynamics model using volatile pressure and squeeze flow during tape placement process. The void motion and filling are simulated using a non-local model where their presence is reflected in the global macroscale behavior. Local pressure gradients during compression do play a critical role in void dynamics, and hence the need for a non-local model. Deriving a non-local model accounting for all the void motion and dynamics entails a prohibitive number of degrees of freedom, leading to unrealistic computation times with classical solution techniques. Hence, Proper Generalized Decomposition - PGD - is used to solve the aforementioned model. In fact, PGD circumvents the curse of dimensionality by using separated representation of the space coordinates. For example, a 2D problem can be solved as a sequence of $1 \mathrm{D}$ problems to find the $2 \mathrm{D}$ solution. The non-local model solution sheds light on the fundamental of the void dynamics including their pressure variation, motion and closure mechanisms. Finally, a post treatment of the transient compression of the voids is used to derive conclusions regarding the physics of the void dynamics.
\end{abstract}

Keywords Void filling · Void dynamics · Tape placement · Proper Generalized Decomposition · PGD $\cdot$ Squeeze flow

\section{Introduction}

Autoclave manufacturing of composite materials although well established is prohibitively expensive and not suitable to make too large and thick parts. Active efforts are underway to find autoclave replacement, with new out-ofautoclave manufacturing processes to improve reliability and production volumes [13, 14, 26]. One of the promising out-of-autoclave composite manufacturing processes is the automated tape placement process (ATP). ATP builds the bulk of a composite part through laying up composite thermoplastic prepregs tape layer after layer over a tool surface. Consolidation of newly added layers is achieved by heating the freshly added tape and compressing the softened

Chady Ghnatios

cghnatios@ndu.edu.lb

1 Department of Mechanical Engineering, Notre Dame University-Louaize, Zouk Mosbeh, PO Box 72, Lebanon

2 University of Delaware, Newark, DE 19716-3119, USA

3 PIMM Laboratory \& ESI GROUP Chair, ENSAM ParisTech, 151 boulevard de l'Hopital, 75013, Paris, France resultant material with a roller to bond it to the previously consolidated layer.

Incoming tape does contain voids and may introduce additional voids due to release of entrapped volatiles and moisture during the heating of the tape, or because the imperfect consolidation of rough tape surfaces as discussed and simulated in $[2,16-19,23]$. Voids are detrimental to the final mechanical properties of the part, hence it is important to address the thermal modeling of the heat process as well as mechanical simulation of the squeeze flow $[6,11]$. Others have modeled the void closure in the ATP process using a coupled thermal mechanical simulation, while using a micro-macro modeling of the void closing either while considering the fiber bed and the matrix as a Newtonian or Non Newtonian mixture and compared it with experimental results $[21,22,27]$. However, the micro-macro modeling of the flow does not allow for void motion, nor does it account for a correct mass balance between the voids in the micro scale model and the bulk material in the macro scale model. Moreover, these models developed at the end of the 20th century are the current state of the art to describe void dynamics $[15,25,28]$. Recently, a new model of void dynamics in multiphase flow, coupling multiple physics at different scales and using optimization algorithms for convergence was derived in [4]. However its application to 
a real composite material part would be complicated and cumbersome.

Moreover, using some simplification while conserving general physical laws, a 1D non-local (non-local in the sense that the pressure driving the voids evolution depends on all the voids in the domain) void closing model was successfully used in [24], without allowing any void motion. From previous studies, and to the best of our knowledge, accounting for void motion and void filling using a nonlocal model has not been addressed until now. Thus, the aim of this work is to model and simulate the void filling and motion from a non-local point of view, using gas pressure inside the void and viscous resin squeeze flow analysis.

A numerical simulation of a non-local void filling and motion model will involve a large number of degrees of freedom leading to infeasible computational time. Therefore, the use of model order reduction techniques was adopted to overcome this hurdle. Model order reduction techniques tend to reduce simulation time without degrading the thermomechanical model [11]. Moreover, the Proper Generalized Decomposition or PGD, is the only "a priori" model order reduction technique able to handle separated space coordinates [7]. Thus, using PGD, a 2D problem can be solved as a sequence of $1 \mathrm{D}$ problems for example, leading to a dramatic reduction in computational time [5, 12]. The use of PGD in composite materials modeling has led to multiple successful simulations of different manufacturing processes $[1,9,10]$. The use of PGD in this work makes it possible to increase the number of nodes to achieve mass balance in the domain and account for void motion.

In this paper, first we present the void dynamics model. Next, we review the PGD algorithm and recast it to address the ATP process. Finally, we show the results for multiple examples while describing the observed physical behavior to gain insight of the process at local level.

\section{Void dynamics model}

In this modeling effort, we consider a tape cross section under the roller as illustrated in Fig. 1. The effect of the temperature change is neglected in this work since the process is taking place in a very small time interval with an almost constant uniform temperature in the entire domain. The boundary conditions are given by the following equations:

$$
\left\{\begin{array}{l}
\mathbf{v}(y=h)=(0,-u) \\
\mathbf{v}(y=0)=\mathbf{0} \\
P(x=0)=P_{a t m} \\
P(x=L)=P_{a t m} \\
P(\text { Void } i)=P_{i}
\end{array}\right.
$$

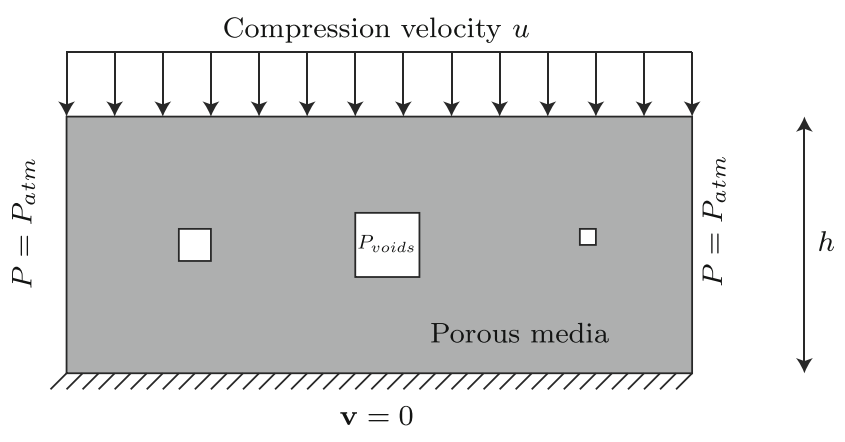

Fig. 1 The considered tape cross section

We assume that the roller compresses the tape from the top with a constant velocity $u$. As ATP is a very fast process, the roller makes contact with the tape for a very short duration. We assume the bottom of the tape does not move, hence zero displacement at the bottom of the domain and atmospheric pressure around the tape. The tape domain may contain multiple voids. Inside a void $i$, the pressure is $P_{i}$ and will be a function of time $t$. For a constant compression velocity under the roller, all cross sections shall undergo the same compression profile, and therefore simulating the compression of one cross section is sufficient to model the void closure process inside the tape.

In this work, we focus on the behavior of void dynamics in a given cross section. Moreover, due to the reduced tape thickness, we neglect any thermal coupling with the fluid viscosity. Readers interested in thermal fields simulation during ATP may refer to $[3,11,20]$.

\section{Governing equations}

In this model, the squeeze flow is the main driving phenomenon that leads to filling the voids. However, the volatile pressure inside the void increases with deceasing void volume and therefore resists the filling of the void with the resin. Since the compression time interval is in general too short and exhibits large pressure gradients, we can safely neglect the capillary and inertia effects. Moreover, outside the void regions, the polymer flow in the composite material is modeled using Darcy's law:

$\mathbf{v}=-\frac{\mathbf{K}}{\mu} \cdot \nabla P$

where $\mathbf{v}$ being the velocity field, $P$ the resin pressure, $\mathbf{K}$ the fiber bed effective permeability and $\mu$ the resin viscosity. Combining Eq. 2 with conservation of mass:

$\nabla \cdot \mathbf{v}=0$ 
results in the strong form of the governing equation inside the porous medium:

$-\nabla \cdot\left(\frac{\mathbf{K}}{\mu} \nabla P\right)=0$

Solving Eq. 4 one can obtain the pressure field inside the tape domain. From the obtained pressure field, we can use Eq. 2 to find the velocity field inside the domain. The volumetric flow rate $Q_{i}$ entering a void $i$ at time $t$ is therefore defined by:

$Q_{i}=-\int_{A_{i}} \mathbf{v} \cdot d \mathbf{A}$

Where $A_{i}$ is the void $i$ surface at time $t$ and $d \mathbf{A}$ is a differential surface element of void $i$ pointing towards the resin domain.

However Eq. 4 doesn't account for the presence of the voids inside the simulated domain, and the resultant changes in the corresponding pressure field. Thus, a change in the formulation inside the voids is mandatory to define a non-local void compaction model.

\section{Void model}

First of all, we assume no reaction occurs between the voids and the surrounding medium. As a result, inside the voids, the ideal gas law drives the pressure's behavior. We can define the rate of change of the volume of void $i$ by $\dot{V}_{i}$ as:

$\dot{V}_{i}=-Q_{i}=\int_{A_{i}} \mathbf{v} \cdot d \mathbf{A}$

Using the ideal gas law on void $i$ at time $t$, we can write:

$\dot{P}_{i}=-\dot{V}_{i} \cdot \frac{P_{i}}{V_{i}}$

We can therefore upgrade void $i$ pressure and volume from time $t_{n}$ to time $t_{n+1}$ using Euler's approximation of the derivatives leading to:

$\left\{\begin{array}{l}V_{i}^{n+1}=V_{i}^{n}+\dot{V}_{i} \cdot \Delta t \\ P_{i}^{n+1}=P_{i}^{n}+\dot{P}_{i} \cdot \Delta t\end{array}\right.$

where $\Delta t$ is the considered time step.

The pressure inside the voids is considered as an imposed Dirichelet boundary condition. Therefore, considering the high pressure induced by the squeeze flow, the pressure gradients would increase dramatically in the void neighborhood. To capture these pressure gradients accurately, a highly refined mesh is required, involving a prohibitive number of degrees of freedom. This leads to infeasible computation time, which is the main hurdle to the use of this non-local void models. Therefore, model order reduction techniques especially PGD is a suitable candidate for the aforementioned model.

\section{PGD simulation}

Model order reduction techniques are advanced simulation techniques using domain decomposition into lower dimensionality domains. For example our 2D problem can be solved as a sequence of 1D problems as illustrated in Fig. 2. The approach provides the exact full dimensionality solution, but with reduced computational cost. In this section we review the PGD algorithm used in this work and the integration of the boundary conditions introduced by the presence of voids in the domain.

\section{The PGD algorithm}

The PGD algorithm starts by writing the integral form of the problem governing Eq. 4:

$-\int_{\Omega} P^{*} \nabla \cdot\left(\frac{\mathbf{K}}{\mu} \cdot \nabla P\right) d \Omega=0$

where $P^{*}$ is the test function. Later on, we define $P$ in a separated form as a series such as:

$P \approx \sum_{j=1}^{j=N} X_{j}(x) Y_{j}(y)$

where $X_{j}$ and $Y_{j}$ are not defined "a priori" but found by the algorithm. In fact we suppose that $P$ is known until the term $l$ and seek to find the next term of the sequence represented as:

$P_{l+1}=\sum_{j=1}^{j=l} X_{j}(x) Y_{j}(y)+R(x) S(y)$
Fig. 2 Domain decomposition: a problem in the $(x ; y)$ domain is solved as a sequence of $x$ then $y$ problems

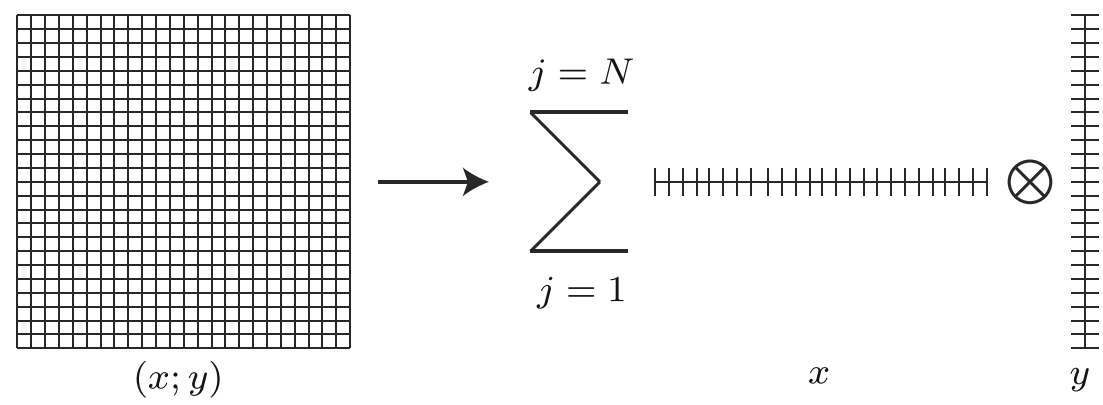


$R(x)$ and $S(y)$ being the enrichment terms, leading to $X_{l+1}(x)$ and $Y_{l+1}(y)$ after convergence. Interested reader may refer to [8] and the references therein. We also suppose that the test function $P^{*}$ can be written in a separated form such as [7]:

$P^{*}=R^{*}(x) S(y)+R(x) S^{*}(y)$

where $R^{*}$ and $S^{*}$ are respectively the test functions in the $x$ and $y$ domains.

Substituting Eqs. 12 and 11 into the integral form of Eq. 9, results in the following non linear equation:

$$
\begin{aligned}
& -\int_{\Omega}\left(R^{*}(x) S(y)+R(x) S^{*}(y)\right) \nabla \\
& \cdot\left(\frac{\mathbf{K}}{\mu} \cdot \nabla\left(\sum_{j=1}^{j=l} X_{j}(x) Y_{j}(y)+R(x) S(y)\right)\right) d \Omega=0
\end{aligned}
$$

where $R$ and $S$ are the unknowns of the problem. To solve the non linear problem resulting from Eq. 13, we use a rank one update iterative fixed point algorithm. For instance, we may first consider $R$ as known, and thus set the corresponding test function $R^{*}$ to zero, which reduces Eq. 13 to a $1 \mathrm{D}$ problem where $S$ is the only unknown. The resulting problem can be solved using any classical numerical technique such as the finite elements or the finite differences for example. Second, since $S$ is computed, we may consider $S$ as known and thus set $S^{*}$ to zero, which leads to a $1 \mathrm{D}$ problem giving $R$. The iterative calculation continues until convergence of the product $R S$ is reached. The PGD algorithm is illustrated in Fig. 3. The resulting algorithm reduces the calculation domain from a $2 \mathrm{D}$ problem into a sequence of $1 \mathrm{D}$ problems.

The resulting problems are solved using homogeneous boundary conditions, while setting a first product of

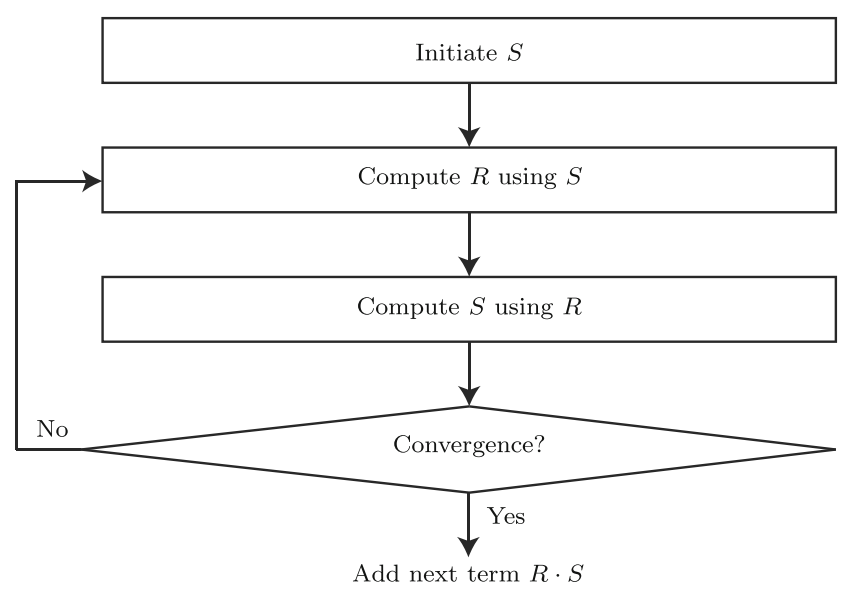

Fig. 3 PGD algorithm used to find the solution of the pressure field functions $X_{1}(x) Y_{1}(y)$ satisfying the non homogeneous essential Dirichlet boundary conditions defined by:

$P=P_{\text {atm }}$ at $x=0$ and $x=L$

with the Neuman ones applying directly in the weak form after integrating by parts.

\section{Treatment of the voids}

The voids, here assumed having a square shape, are randomly distributed along the domain, and therefore the boundary conditions can't be written in the form of a boundary condition in the $x$ domain nor a boundary condition in the $y$ domain. This type of boundary condition should be imposed using a penalty formulation in the PGD separated representation framework [8], by enforcing the pressure multiplied by the penalty acting at the voids locations, the last represented from their characteristic function:

$\chi_{i}=\mathcal{M}_{i}(x) \mathcal{N}_{i}(y), i=1, \cdots, m$,

where $m$ is the total number of voids, and $\mathcal{M}_{i}(x)$ and $\mathcal{N}_{i}(y)$ the characteristic functions in the $x$ and $y$ defining the $\mathrm{i}$-void where penalty will apply:

$\left\{\begin{array}{l}\mathcal{M}_{i}(x)=1 \text { if } x \in \mathcal{V}_{i} ; \mathcal{M}_{i}(x)=0 \text { elsewhere } \\ \mathcal{N}_{i}(y)=1 \text { if } y \in \mathcal{V}_{i} ; \mathcal{N}_{i}(y)=0 \text { elsewhere }\end{array}\right.$

$\mathcal{V}_{i}$ being the part of the computation domain $\Omega$ included in void $i$. With $P_{i}$ denoting the pressure in void $i$ and $\lambda$ the penalty coefficient assumed large enough, the penalized integral form of the problem becomes:

$-\int_{\Omega} P^{*} \nabla \cdot\left(\frac{\mathbf{K}}{\mu} \cdot \nabla P\right) d \Omega+\lambda \sum_{i=1}^{i=m}\left(P-P_{i}\right) \mathcal{M}_{i} \mathcal{N}_{i}=0$.

At each time step $n$, the pressure is solved using the illustrated PGD algorithm, the flow inside the void is computed using the void Eq. 5. Once the velocity near each void $i$ is available, $\dot{V}_{i}$ and $\dot{P}_{i}$ are computed using Eqs. 6 and 7 respectively. Finally we update the void size and pressure using Eq. 8 before going to the next time step $n+1$.

\section{Void dynamics results and discussion}

In this section we illustrate the studied cases of void dynamics using the PGD framework. First of all we illustrate a study of a simple domain with 3 voids, later on we elaborate to a case of 10 voids in a domain at different position in the thickness direction. For the sake of simplicity and without loss of generality, we will consider the fluid viscosity $\mu=1$ Pa.s for all the examples discussed. We 


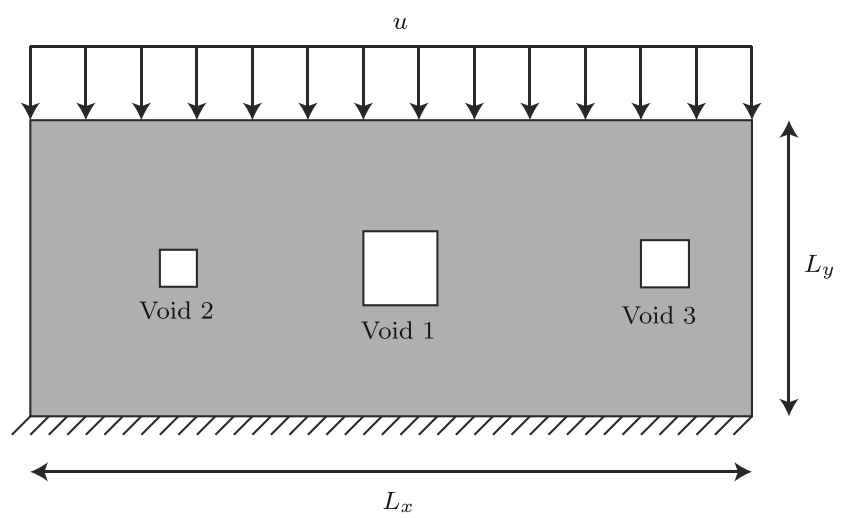

Fig. 4 The considered simple domain (in which Lx and Ly are the width and the thickness of the tape respectively) containing three voids of different sizes. Initially Void 1 is $1.5 \times 1.5 \mathrm{~mm}^{2}$, voids 2 is $1 \times 1 \mathrm{~mm}^{2}$ and void 3 is $1.25 \times 1.25 \mathrm{~mm}^{2}$ in the first example illustrated in Section 3

also consider the voids to be square as the implementation is in cartesian coordinates and as we do not address the surface tension issue, the shape of the void should be of little consequence. Later on, we compare the effect of the void's position and size using the same total initial void volume fraction, on the reduction of void fraction due to squeeze flow induced by the roller during the ATP process. Ellipticity and the associated Saint Venant principle leads to a pressure field that does not depend on the local effects when we move away from the voids. Finally, in this section we aim to identify the effect of void distribution and size on the final void volume fraction.

\section{A simple 3 voids case}

In this section we illustrate a simplified case with 3 voids of different sizes, compressed for two different tape thicknesses, a thick tape and a thin tape. The simulated domain is illustrated in Fig. 4.

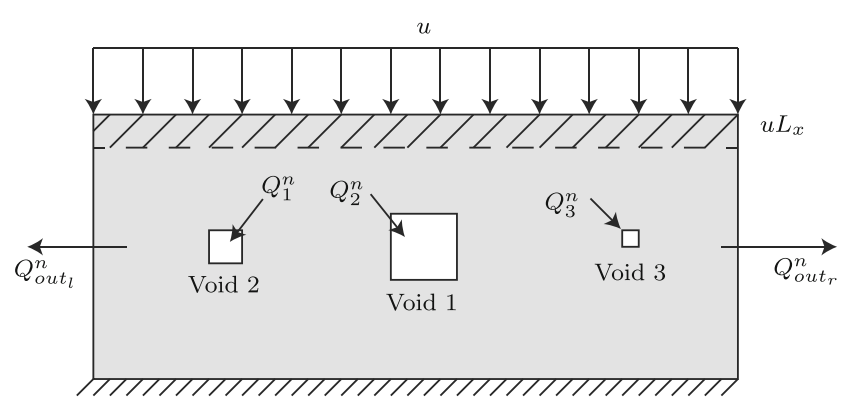

Fig. 5 The mass conservation balance at a given time step $n$ : the flow going in the voids and out of the domain is equal to the displaced volume

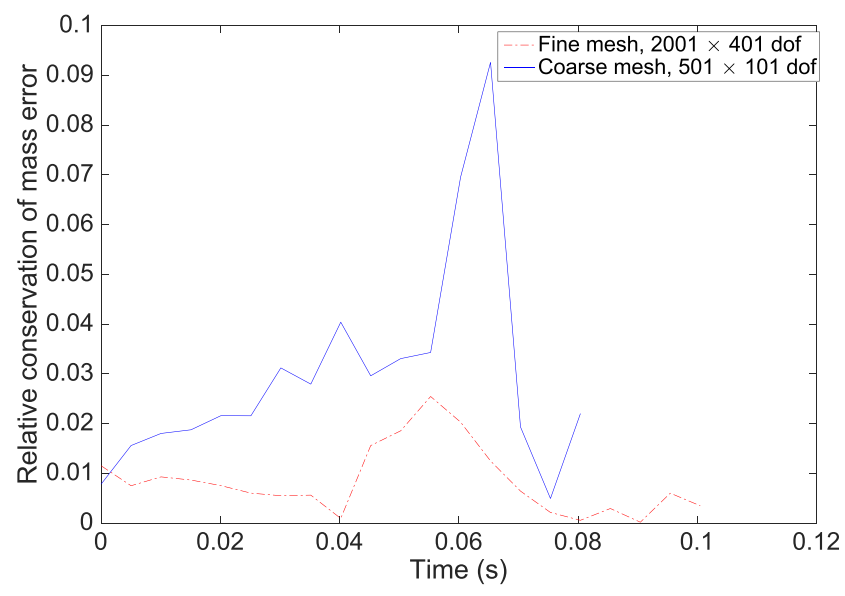

Fig. 6 Error on the conservation of mass using two different meshes with 300 PGD products of functions

\section{A test domain}

First of all we illustrate the results of the model on a domain of dimensions $L_{x}=10 \mathrm{~mm}$ (width of tape under the roller) $\times L_{y}=0.5 \mathrm{~mm}$ (thickness of tape). The chosen compression velocity is set to $u=0.1 \mathrm{~mm} / \mathrm{s}$ along the $y$ direction, as illustrated in Fig. 4. The considered permeability in this section of the tape containing fibers and resin in the length direction is $\mathbf{K}=10^{-12} \mathrm{~m}^{2}$. In this section, the time step is set to be $\Delta_{t}=5 \mathrm{~ms}$. Moreover, to estimate the error on the results, we use an error estimator based on the conservation of mass. For instance, the relative conservation of mass error $\mathcal{E}_{n}$ at an instance $t_{n}$ can be considered as the difference between the flow into the voids and the flow out of the domain from two sides, and the compressed volume from

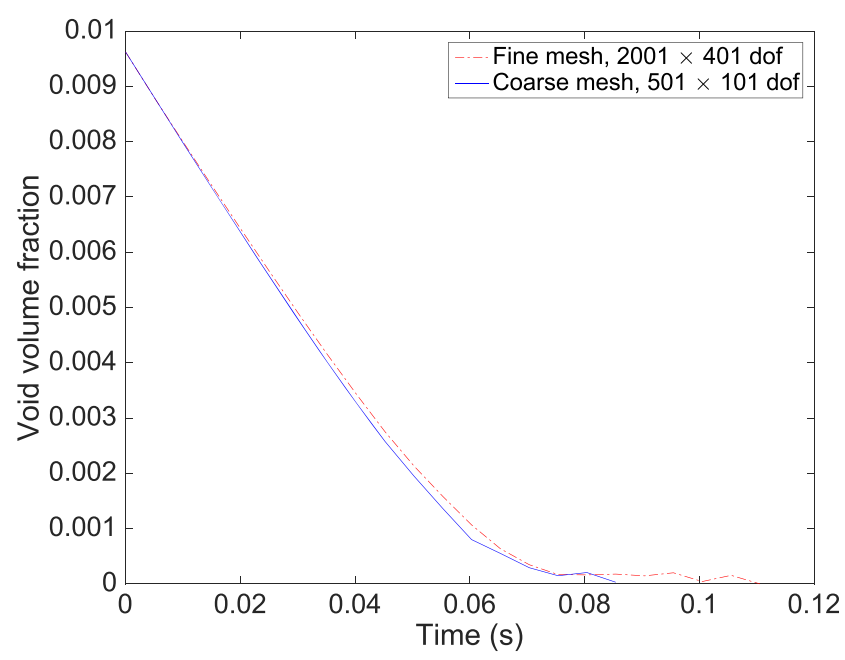

Fig. 7 Total void volume fraction using two different meshes 
the top as illustrated in Fig. 5 and expressed by the following equation:

$\mathcal{E}_{n}=\sqrt{\frac{\left(u L_{x}-\left(\sum_{i=1}^{i=m} Q_{i}^{n}+Q_{\text {out }}^{n}\right)\right)^{2}}{\left(u L_{x}\right)^{2}}}$

where $m$ is the number of voids in the domain at an instant $t_{n}, m=3$ in this example. $Q_{i}^{n}$ is the flow rate into a void $i$ at the instant $t_{n}$ and $Q_{\text {out }}^{n}$ the flow rate out of the domain at an instant $t_{n}$ defined by:

$Q_{\text {out }}^{n}=\int_{x=-L_{x} / 2} \mathbf{v}_{n} \cdot d \mathbf{A}_{n}+\int_{x=L_{x} / 2} \mathbf{v}_{n} \cdot d \mathbf{A}_{n}$

The error $\mathcal{E}$ is plotted as a function of time in Fig. 6 for two different meshes. We can notice that the relative conservation of mass error does not exceed $2.5 \%$ at any time step for the mesh using $2001 \times 401$ nodes, equivalent to 802401 degrees of freedom in 2D. The error doesn't improve anymore with mesh refinement. Moreover, Fig. 7 shows the void volume fraction for both meshes, where the difference between both meshes is not of much relevance. Therefore, for the rest of the work a mesh of $2001 \times 401$

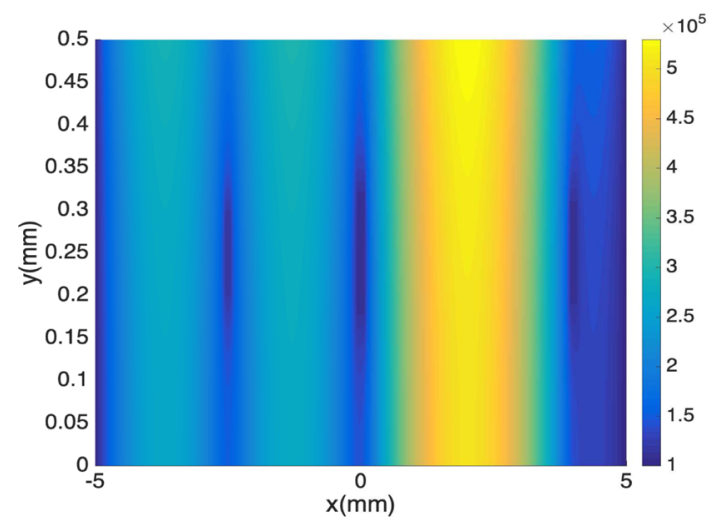

(a) $t=10 \mathrm{~ms}$

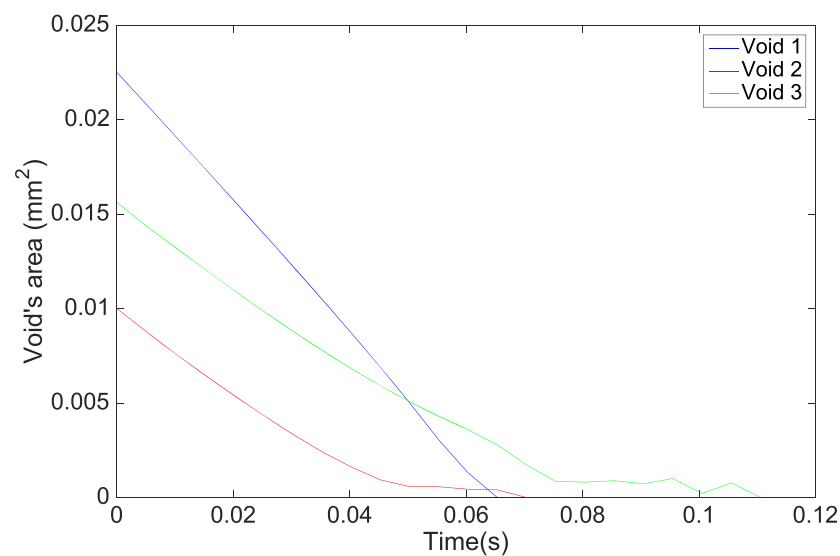

Fig. 9 Change of the in-plane area $\left(\mathrm{mm}^{2}\right)$ of the three voids as a function of the time $(s)$

nodes is adopted. The void volume fraction is computed using:

$v_{f}=\frac{\sum_{i=1}^{i=m} V_{i}}{V_{n}}$

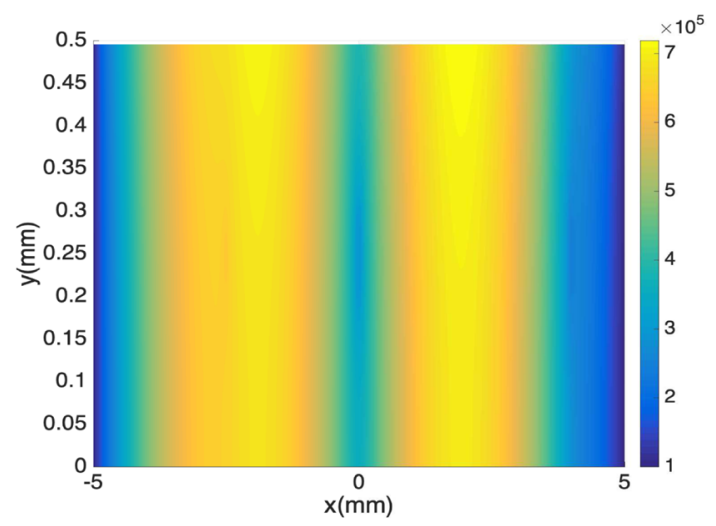

(b) $t=50 \mathrm{~ms}$

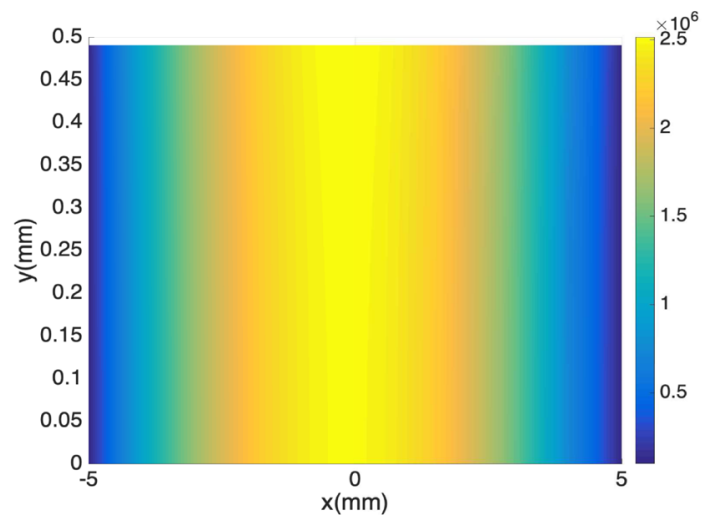

(c) $t=100 \mathrm{~ms}$

Fig. 8 Pressure fields $(\mathrm{Pa})$ inside the considered test domain at different timesteps 


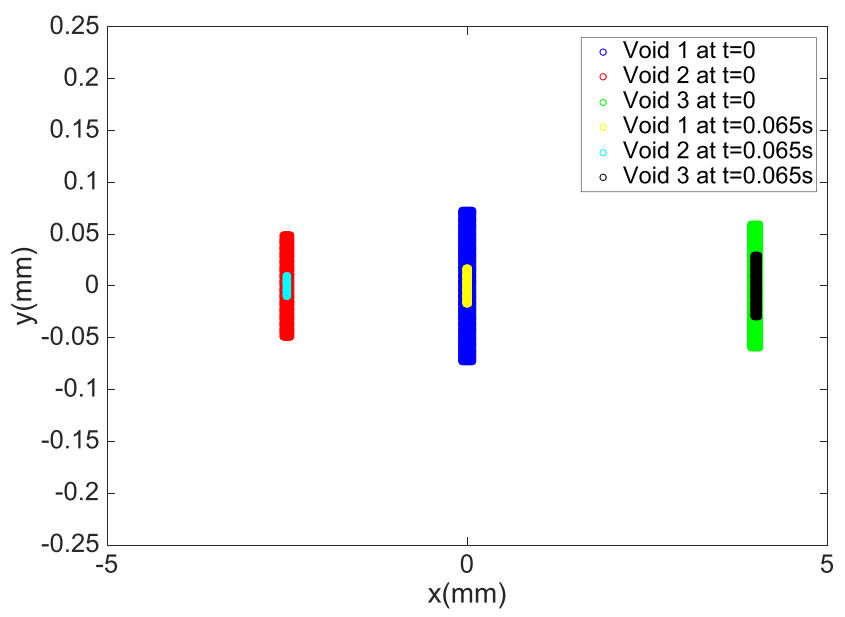

Fig. 10 Compression of the initial voids at three different location at $t=0.065 \mathrm{~s}$. The inital voids at $t=0 \mathrm{~s}$ are the bigger ones, and the smaller ones are the voids at $t=0.065 \mathrm{~s}$

Where $V_{i}$ is the area of void $i$ and $V_{n}$ is the total cross sectional area at instant $t_{n}$.

Using the $2001 \times 401$ mesh, we illustrate the domain pressure at three different time steps $t=10 \mathrm{~ms}, t=50 \mathrm{~ms}$

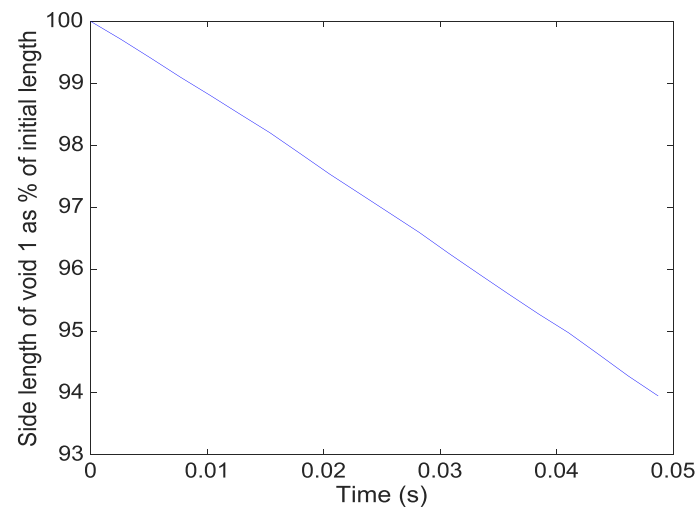

(a) Void 1 and $t=100 \mathrm{~ms}$ in Fig. 8a, b and c respectively. On the other hand, we can clearly identify the increase in the pressure field during compression and void closure. The simulation is stopped once all the voids are closed. A void is supposed dissolved in the matrix and therefore totally closed once its size is smaller than 2 mesh elements.

The center of the void initial positions were set to $x_{1}=$ $0, x_{2}=-0.25 L_{x}$ and $x_{3}=0.4 L_{x}$ for voids 1,2 and 3 respectively. Figure 9 shows the change in the size of the void as a function of time $t$. Figure 9 clearly shows a reduction in void area as a function of time, with a higher rate in the middle of the domain as the pressure is higher in the middle of the domain. Void 3 takes more time to dissolve into the matrix, as it is further away from the center and therefore experiences a lower applied pressure.

We can also compute the horizontal motion of the center of the voids by identifying the velocity difference between the left side and the right side of the voids. Same way we can compute the vertical displacement of the voids centers by using the vertical velocity on the top and bottom of the voids.

We can identify the highest displacement is the one of void 3 , since it exhibits the larger velocity difference

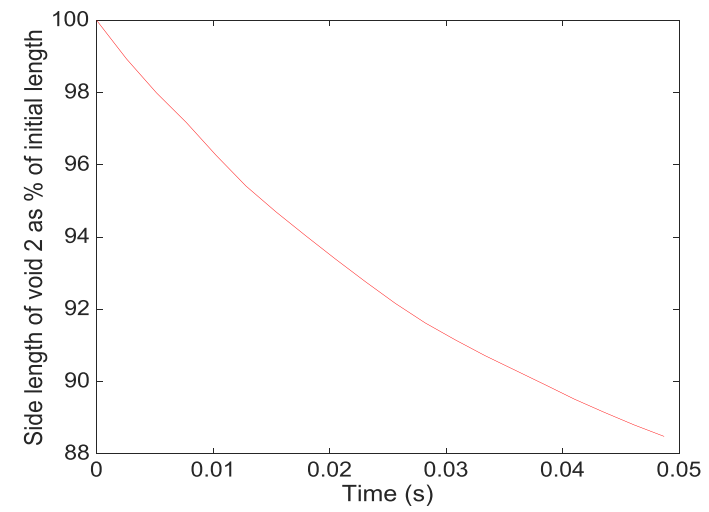

(b) Void 2.

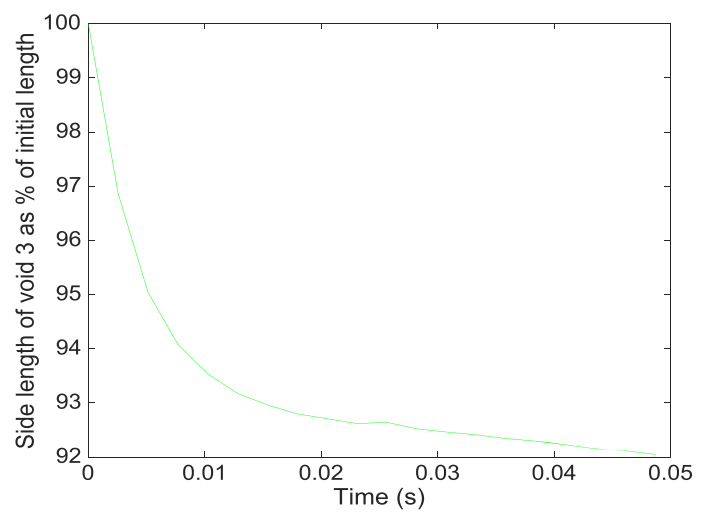

(c) Void 3.

Fig. 11 Square voids side length as a function of the time 


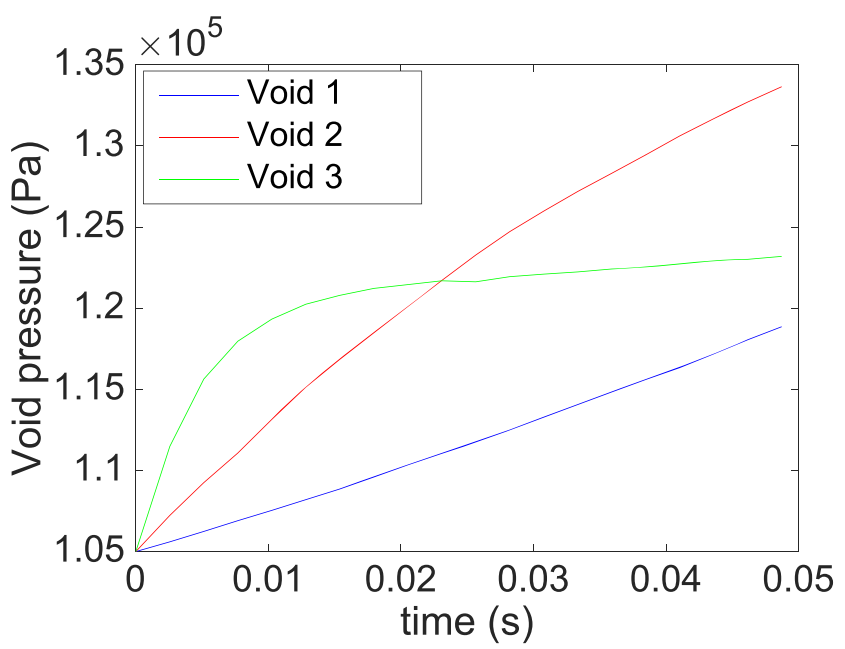

Fig. 12 Pressure fields inside the voids as a function of the time

between the left and the right side of the void. As for the displacement in the vertical direction, a linear displacement downward as a function of the time $t$ is observed for the three voids.

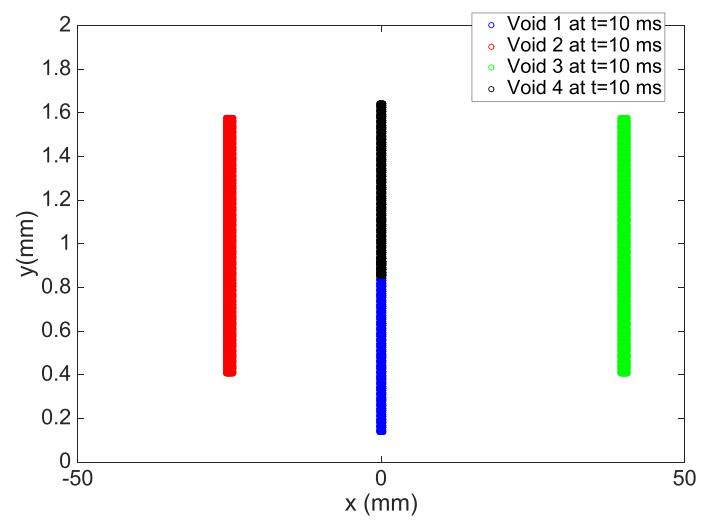

(a) $t=10 \mathrm{~ms}$
The void positions and sizes, at $t=0 \mathrm{~s}$ and $t=0.065 \mathrm{~s}$ are illustrated in Fig. 10. We can clearly identify a change of dimensions of the voids.

\section{A thick test domain}

In this section, the illustrated results are shown on a domain of dimensions $L_{x}=100 \mathrm{~mm} \times L_{y}=10 \mathrm{~mm}$ compressed at a velocity of $u=0.1 \mathrm{~mm} / \mathrm{s}$ along the $y$ direction as illustrated in Fig. 4. We consider a permeability $\mathbf{K}=10^{-10} \mathrm{~m}^{2}$ and a time step $\Delta t=2.6 \mathrm{~ms}$.

We plot the size of the three voids as a function of the time in Fig. 11. Figure 12 shows an increase in the voids pressure as a function of the time, however at a different pace from one void to another. In fact, void 1 is the largest void initially, which means it can absorb the most fluid before reaching equilibrium with its environment, while void 3 absorbs the least resin before equilibrium. Void 3 therefore reaches equilibrium, which means a stagnation in pressure and void size as shown in Figs. 12 and 11c respectively, while void 1 is still decreasing its size and increasing its pressure quasi linearly as a function of the

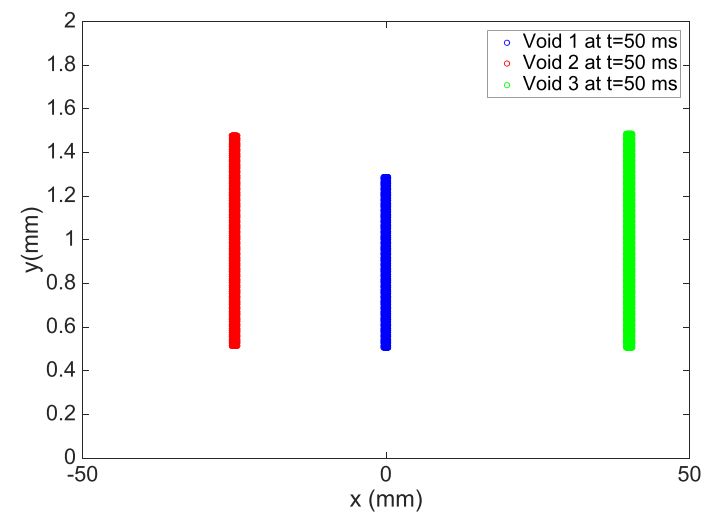

(b) $t=50 \mathrm{~ms}$

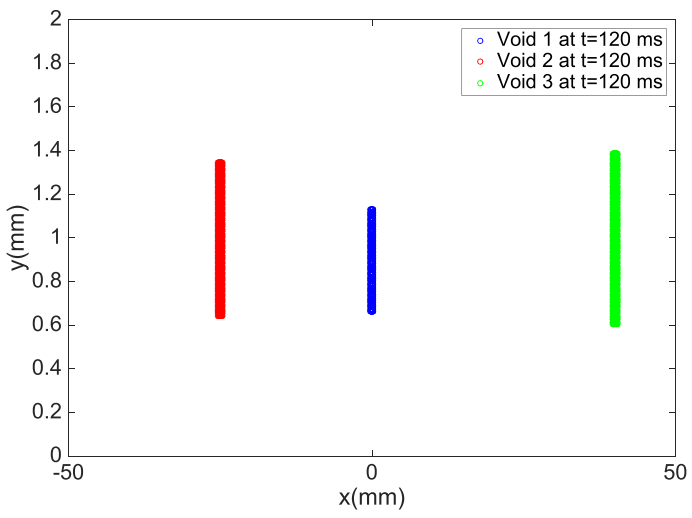

(c) $t=120 \mathrm{~ms}$

Fig. 13 Merging two overlapping voids, compression at $u=0.1 \mathrm{~mm} / \mathrm{s}$ 


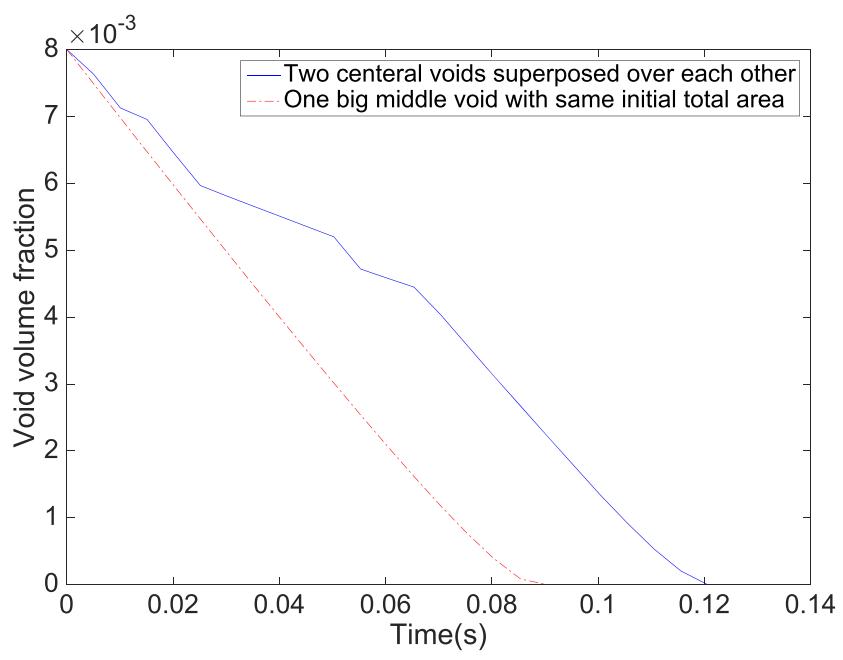

Fig. 14 Void volume fraction $v_{f}$ for a single void and 2 merging voids

time, as shown in Figs. 11a and 12 respectively. Void 2 has an initial dimension between void 1 and 3 as shown in Fig. $11 \mathrm{~b}$, therefore the rate of pressure change in void 2 is decreasing at the end of the simulated time interval, but still has not reached equilibrium with its environment. Note that the pressure in void 2 is apparently larger than the pressure in void 1 at $t=0.05 \mathrm{~s}$, however this is not really relevant since the pressure in void 1 will continue to increase until reaching its environment pressure, which means eventually a pressure larger than void 2 since the pressure in the middle of the domain is definitely larger than the pressure at the edges. Note that the simulation stopped once one of the voids reaches equilibrium with its environment, therefore a volumetric flow $Q_{i} \approx 0$ is registered in that void.

\section{Merging voids}

One may imagine the presence of 2 nearby voids, for instance a void 4 just above void 1 . In case of merging voids,

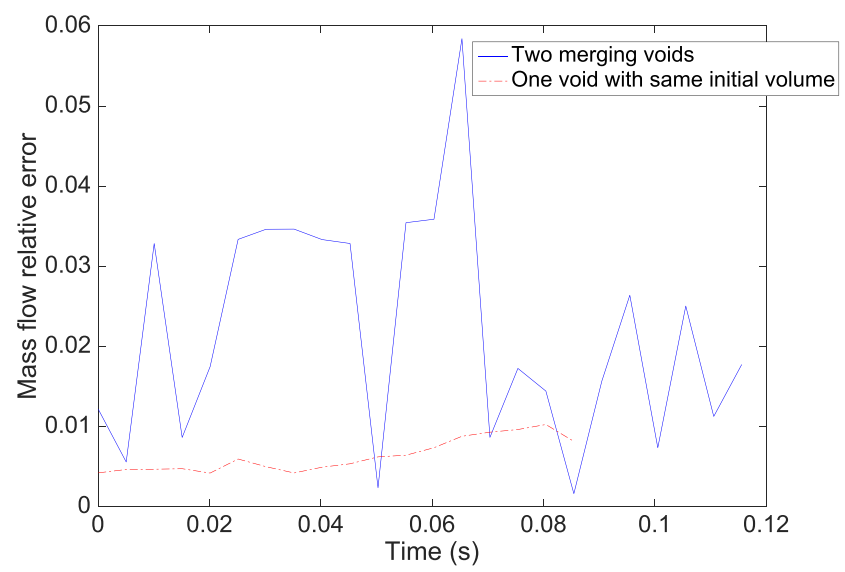

Fig. 15 Relative mass flow error for a single void and 2 merging voids

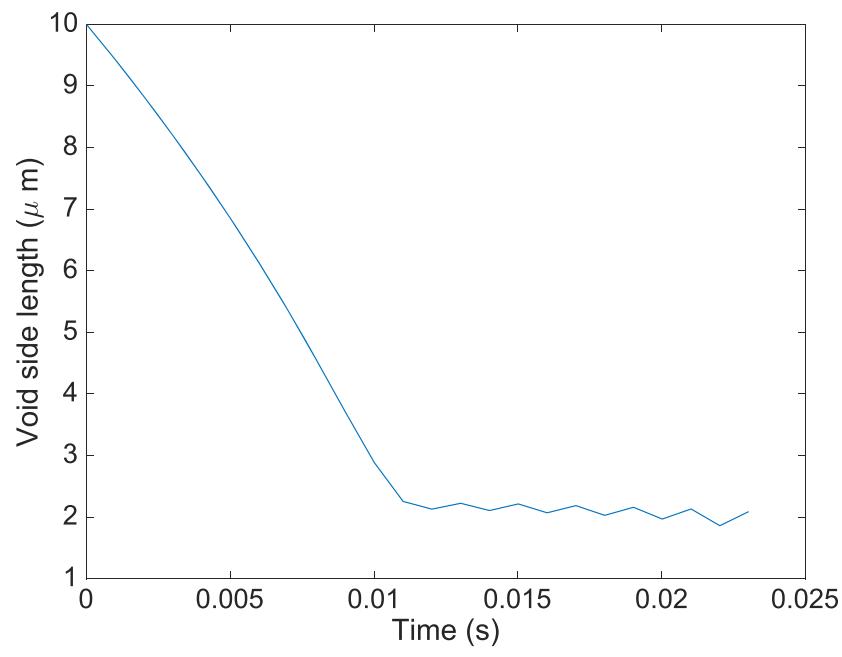

Fig. 16 A void having the orger of magnitude of one $\mu \mathrm{m}$

where the boundaries overlap, we can use basic physical laws to find the final pressure and size of the voids. Using the conservation of mass one may write:

$m_{\text {new }}=m_{i}+m_{j}$,

Where $m_{i}$ and $m_{j}$ are respectively the masses of the two merging voids $i$ and $j$ respectively, while the mass in the newly formed void after merging them is $m_{\text {new }}$. Now using the ideal gas law one may write:

$P_{\text {new }}=\frac{(P V)_{i}+(P V)_{j}}{V_{i}+V_{j}}$

An example of two merging voids, void 1 and 4 for instance, is illustrated in Fig. 13.

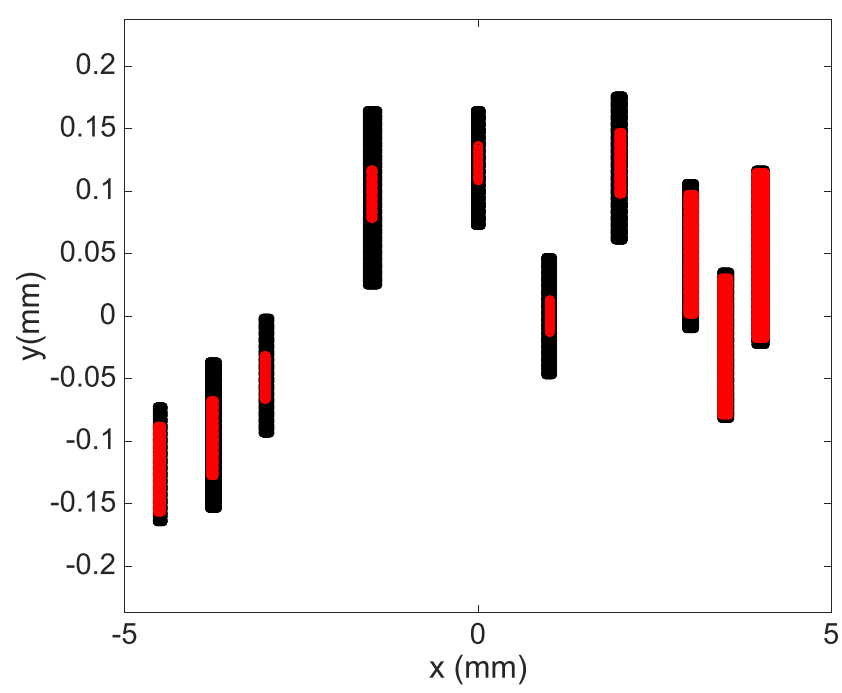

Fig. 17 Initial positions of the void centers, numbered 1 to 10 from the left to the right according to their initial $x$ coordinates at $t=0$. In black the inital distribution at $t=0$ and in red the voids distribution at $t=140 m s$ 
Table 1 Positions and dimensions of the considered voids

\begin{tabular}{|c|c|c|c|c|c|c|c|c|c|c|}
\hline Void number & 1 & 2 & 3 & 4 & 5 & 6 & 7 & 8 & 9 & 10 \\
\hline Initial side length $(\mu m)$ & 100 & 125 & 100 & 150 & 100 & 100 & 125 & 125 & 125 & 150 \\
\hline$x$ coordinate $(\mathrm{mm})$ & -4.5 & -3.7 & -3 & -1.5 & 0 & 1 & 2 & 3 & 3.5 & 4 \\
\hline$y$ coordinate $(\mu m)$ & -125 & -100 & -50 & 100 & 125 & 0 & 125 & 50 & -25 & 50 \\
\hline
\end{tabular}

To compare the void volume fraction reduction as a function of the time between one void and 2 merging voids of the same initial volume, we consider a $0.5 \mathrm{~mm} \times 10 \mathrm{~mm}$ domain of the tape being compressed at a constant velocity $u=0.1 \mathrm{~mm} / \mathrm{s}$. In Fig. 14, we compare the void volume fraction of one central void placed at $x=y=0$, with two voids located at $x=0$ with $y=-0.11 \mathrm{~mm}$ for the fist void and $0.025 \mathrm{~mm}$ for the second one. Figure 15 shows relative error in the conservation of mass for both cases. We can clearly note that closing 2 voids takes more time than closing a single void. We also note that the relative error is higher in the case of two merging voids. This can be explained by the fact that the domain area between the two voids is relatively thin and require a high number of PGD modes to be represented with high precision.

We also note that the voids need to be within close proximity of each other to merge. In our test case, if the voids initially are more than $10 \mu \mathrm{m}$ apart in the vertical direction, the void closing speed overcomes the motion of the boundaries and the voids don't merge.

\section{Microscopic void compression}

In this section we explore the posibility of simulating the compression of voids with a dimension lower than a $\mu \mathrm{m}$.

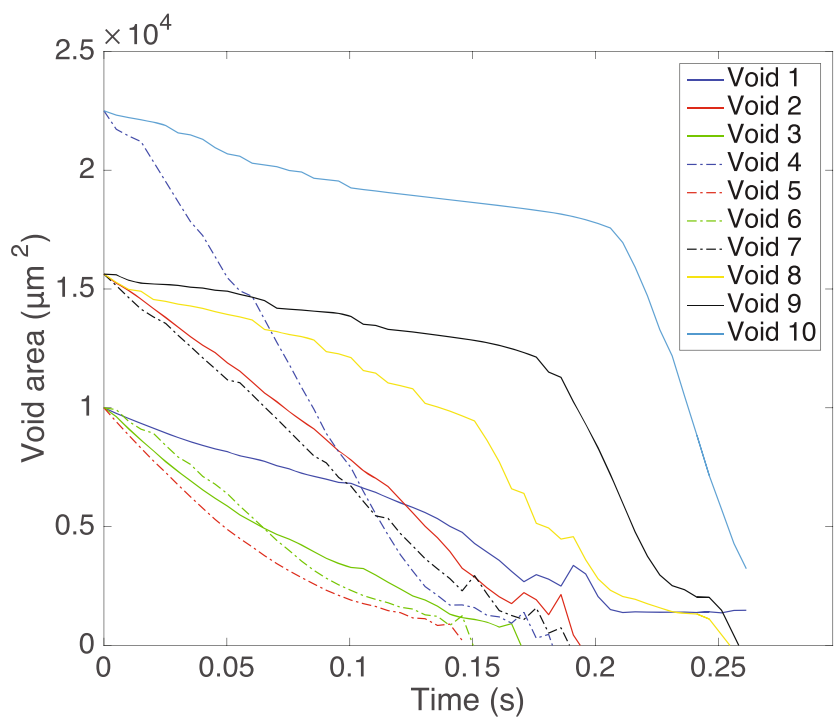

Fig. 18 Voids' area in the simulated domain using $u=0.1 \mathrm{~mm} / \mathrm{s}$ along the $y$ axis
We consider a domain of dimension $4.2 \mathrm{~mm}^{2}$ with a middle void of initial dimension $10 \mu \mathrm{m}$. A timestep of $1 \mu \mathrm{s}$ is used. The results are illustrated in Fig. 16.

We can clearly see that the method has potentially no limit for illustrating voids with dimensions having the order of magnitude of $1 \mu \mathrm{m}$, however for sake of practicality, we will stick to $9 \mu \mathrm{m}$ as a lower limit of the void size for the rest of this work.

\section{A more realistic case}

In this section we compress a more realistic tape initially containing 10 voids of different sizes in a tape domain under the roller of dimensions of $L_{x}=10 \mathrm{~mm}$ and $L_{y}=0.5 \mathrm{~mm}$. We use in this section a permeability of $\mathbf{K}=10^{-12} \mathrm{~m}^{2}$ and a time step of $\Delta t=5 \mathrm{~ms}$. We compress it under $u=0.1 \mathrm{~mm} / \mathrm{s}$ in the thickness direction.

To improve the convergence of the PGD algorithm in this case, we start by initializing a "known" set of functions using the previously calculated modes, in fact we may use:

$P^{n+1}=P_{\text {edited }}^{n}+\sum_{j=1}^{j=N} X_{j}(x) Y_{j}(y)$,

Where $P_{e d i t e d}^{n}$ is equal to $P^{n}$, but changing the voids' pressures $P_{i}^{n}$ into the voids' pressures $P_{i}^{n+1}$. This helps

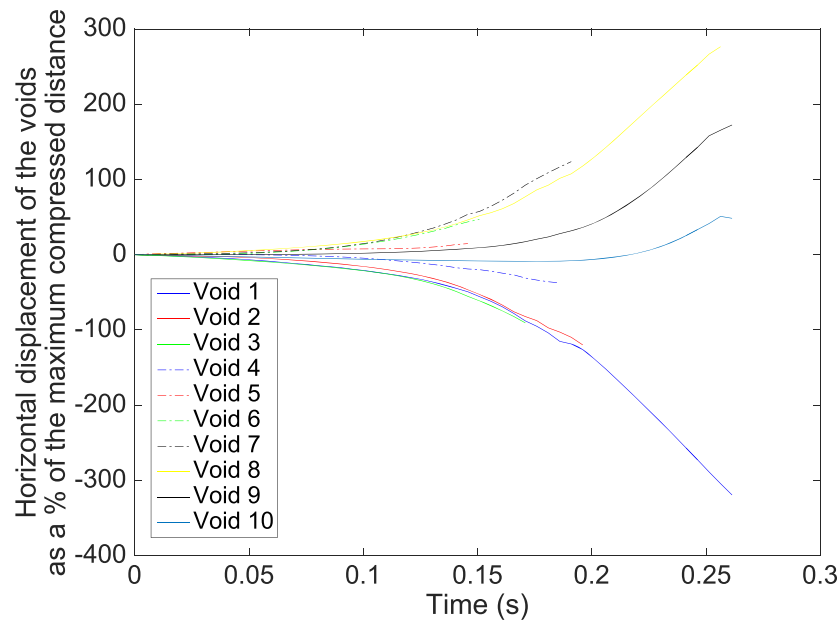

Fig. 19 Voids' displacements in the simulated domain using $u=$ $0.1 \mathrm{~mm} / \mathrm{s}$ along the $y$ axis, as a percentage of the maximum compressed distance, equal to $26.2 \mu \mathrm{m}$ 
with the algorithm convergence and avoids recreating the PGD solution. The considered voids have their centers at the initial positions shown in Fig. 17, and their initial side length and positions are given in Table 1 . The voids are numbered 1 to 10 from the left to the right using their $x$ coordinates. Note that void number 4 is touching the upper surface in our model to test the possibility of compressing surface voids, without allowing it to escape through the upper domain boundary. We may note that in this simulation, a void is considered as totally closed, disappearing therefore from the model, at a lower threshold.

In this section we compress the voids at $u=0.1 \mathrm{~mm} / \mathrm{s}$. We illustrate the voids areas in Fig. 18. We can clearly identify the middle voids (in dashed lines) closing faster than the side voids. This is explained by the higher pressure distribution in the middle of the domain in general. We can

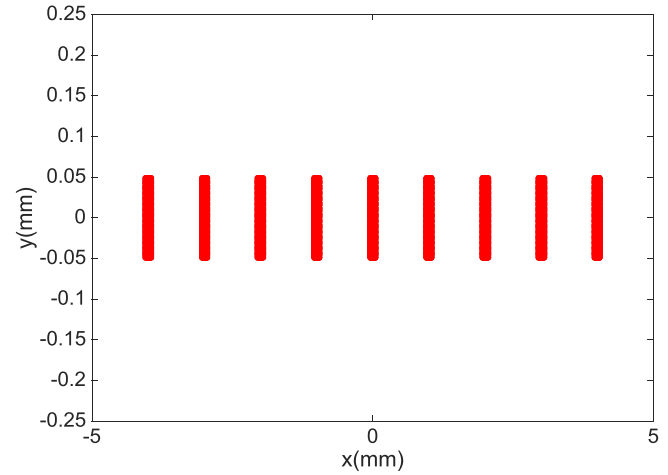

(a) Homogeneous distribution

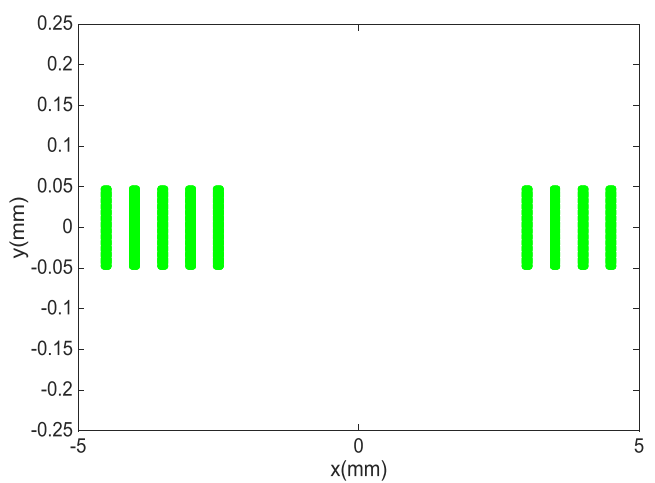

(c) Side concentration

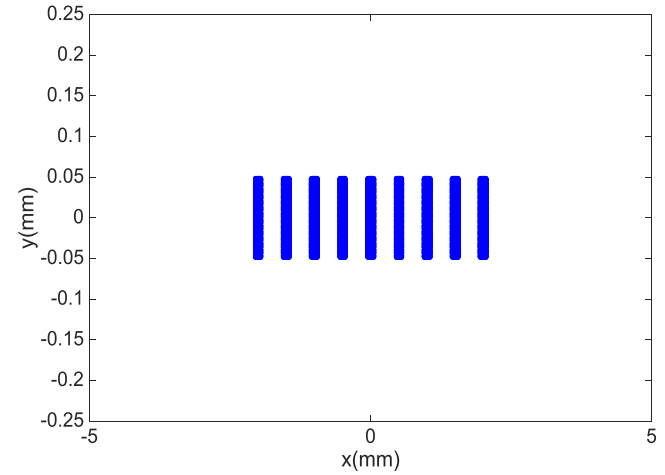

(b) Central distribution

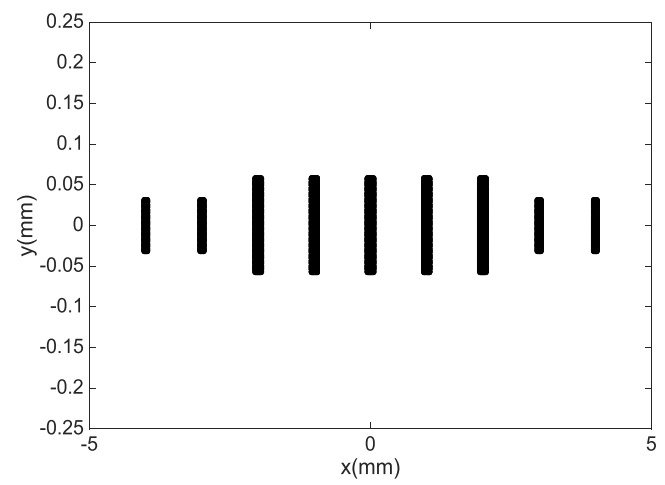

(d) Big central voids with uniform distribution along $x$ direction

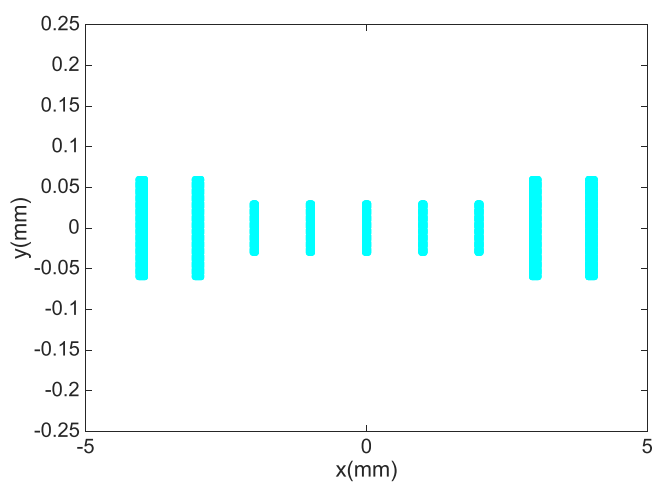

(e) Big side voids with uniform distribution along $x$ direction

Fig. 20 The five tested cases of void distribution 
also deduce an interaction between nearby voids through the reduction of the surrounding pressure. We may also note that equilibrium is reached in some voids.

In Fig. 19 we illustrate the displacement of the voids, as a percentage of the total compressed distance. We can clearly see that the left voids in general move to the left and the right voids move to the right, except for void 10 which moved in the negative direction but at some point switched back to move in the positive direction once again. We note that the maximum dispacement of the voids is about $300 \%$ of the total compressed distance, equal to $26.2 \mu \mathrm{m}$. Thus the maximum horizontal displacement of voids has the order of magnitude of about $80 \mu \mathrm{m}$. Finally the relative error in the conservation of mass as defined in Eq. 18 doesn't exceed $4 \%$ in any time step.

The behavior of void 10 shown in Fig. 18 appears to be interesting in the study of voids' motion interdependency. First of all, we note that voids 8,9 and 10 are very close to each others. The fluid flow would naturally start from the center of the domain (the high pressure region) towards the sides (low pressure regions). Thus, the fluid flow will first fill void 8 , than void 9 , in order to reach void 10 . Void 10 therefore starts closing at an accelerated pace only after the sizes of void 8 , then 9 , are reduced considerably.

\section{Role of void distribution and initial size}

In this section we study the effect of the variation of the position of the voids with respect to the total void volume fraction in the domain. Therefore, we consider nine voids of same initial void volume fraction with five different distributions of voids:

- Uniform distribution illustrated in Fig. 20a.

- Voids concentrated in the middle of the domain, as per Fig. 20b.

- Voids concentrated on the sides of the domain, as shown in Fig. 20c.

- Uniformly distributed voids along $x$-direction with big voids in the center of the domain, as per Fig. $20 \mathrm{~d}$.

- Uniformly distributed voids along $x$-direction with big voids at the domain's edges, as shown in Fig. 20e.

In Fig. 21 we plot the void area fraction $v_{f}$ as a function of the time for the five different distributions. We can clearly identify that an homogeneous distribution of voids would reduce $v_{f}$ to zero the fastest among the tested cases, while concentrated distributions of voids takes more time and even reach a stagnation point after some time. This is also illustrated in Section 3, where voids 8, 9 and 10 were placed near each others and void 10 was the last to considerably change its size. One may note that for the case of voids present only in the center of the domain, the middle voids never closed since it is localized in a region

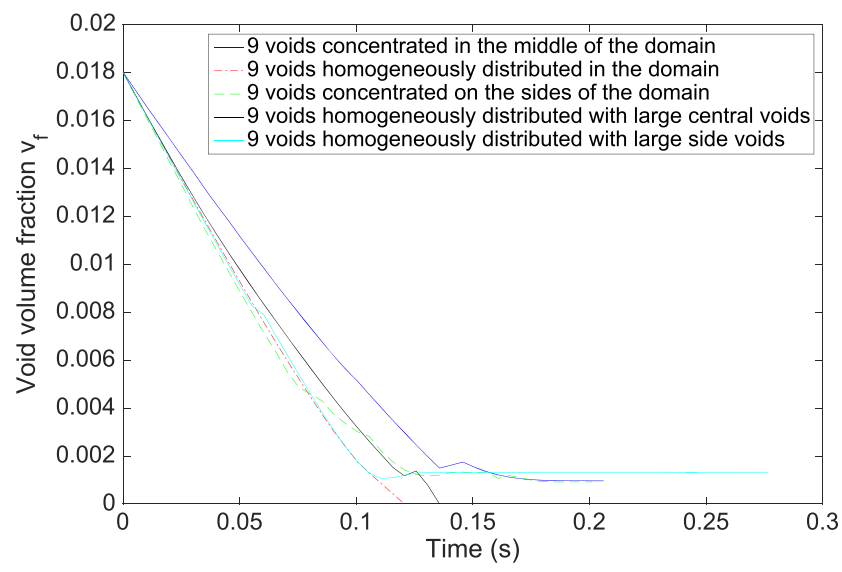

Fig. 21 Void area fraction variation as a function of the time for different voids distributions

of very low pressure gradient. On the other hand, the slight deconsolidation in $v_{f}$ can be attributed to a large time step in the time mesh, where the void pressure increased more than the surrounding local pressure at a given time step, without a major impact on the global solution. From Fig. 21, we can conclude that an homogeneous distribution of voids is easier to close since it allows the highest possible pressure gradients.

On the other hand, the influence of the void dimension is clearly illustrated in Fig. 21. We can note that the presence of large voids decrease the total void volume fraction reduction pace. In fact, larger voids need more time to close than smaller voids. We can also note that the void distribution containing large voids in the middle of the domain reaches a total $v_{f}=0$, but the presence of big voids on the sides reaches a stagnation point. This was also noted in Section 3, where large voids 8 and 9 hinder the fluid flow towards void 10 , which is also one of the largest voids in the considered domain.

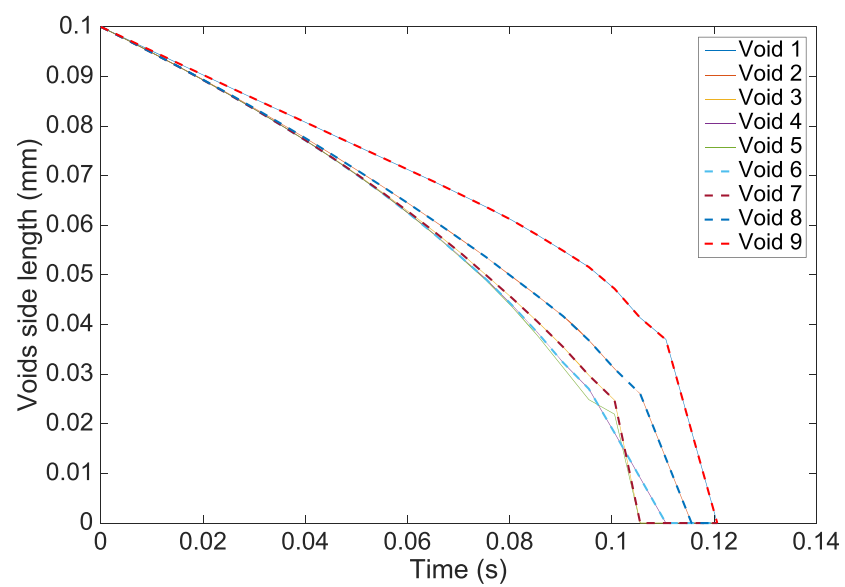

Fig. 22 Voids side length in the homogeneous distribution illustrated in Fig. 20a, numbered 1 to 9 from the left to the right. We see that symmetrical voids around the central axis exhibit similar behavior 
Moreover, the maximum mass conservation error is about $4.5 \%$ for any of the five illustrated cases in this section. We can also illustrate the accuracy of the solution by showing the void variation in the homogeneous domain, illustrated in Fig. 22, where the solution is $100 \%$ symmetrical.

\section{Conclusions}

This work uses a non-local model to simulate the consolidation and void dynamics behavior, while considering the presence of voids in the continuum of the simulated domain. The illustrated model captures the fundamental void dynamics including their pressure variation, motion and closure mechanism. The work introduces PGD formulation to reduce the computational burden to address the void dynamics modeling that may be used in future work to simulate multiple voids consolidation and deduce final microstructure with void sizes and location at the end of the consolidation time period in a ATP process.

\section{Compliance with Ethical Standards}

Conflict of interests The authors declare that they have no conflict of interest.

\section{References}

1. Aguado JV, Borzacchiello D, Ghnatios C, Lebel F, Upadhyay R, Binetruy C, Chinesta F (2017) A simulation app based on reduced order modeling for manufacturing optimization of composite outlet guide vanes. Advanced Modeling and Simulation in Engineering Sciences 4(1): 1-26

2. Argerich C, Ibanez R, Leon A, Barasinski A, Abisset-Chavanne E, Chinesta F (2018) Tape surface characterization and classification in automated tape placement processability: modeling and numerical analysis. AIMS Materials Science 5(5):870-888

3. Barasinski A (2012) Modlisation du procd de placement de fibres thermoplastiques. PhD thesis, Ecole Centrale Nantes

4. Barlow A, Klima M, Shashkov M (2018) Constrained optimization framework for interface-aware sub-scale models of void closure in lagrangian hydrodynamics. J Comput Phys 371:914944

5. Bordeu F, Ghnatios C, Boulze D, Carles B, Sireude D, Leygue A, Chinesta F (2015) Parametric 3d elastic solutions of beams involved in frame structures. Advances in Aircraft and Spacecraft Science 2(3):233-248

6. Bur N, Joyot P, Ghnatios C, Villon P, Cueto E, Chinesta F (2016) On the use of model order reduction for simulating automated fibre placement processes. Advanced Modeling and Simulation in Engineering Sciences 3:1-18

7. Chinesta F, Ammar A, Cueto E (2010) Recent advances in the use of the proper generalized decomposition for solving multidimensional models. Arch Comput Meth Eng 17(4):327-350
8. Chinesta F, Keunings R, Leygue A (2014) The Proper Generalized Decomposition for Advanced Numerical Simulations. Springer, Berlin

9. Chinesta F, Leygue A, Bognet B, Ghnatios C, Poulahon F, Bordeu F, Barasinski A, Poitou A, Chatel S, Maison-Le-Poec S (2014) First steps towards an advanced simulation of composites manufacturing by automated tape placement. Int $\mathbf{J}$ Mater Form $7(1): 81-92$

10. Cueto E, Ghnatios C, Chinesta F, Monte N, Sanchez F, Falco A (2014) Improving computational efficiency in $1 \mathrm{~cm}$ by using computational geometry and model reduction techniques. Key Eng Mater 611:339-343

11. Ghnatios C (2012) Modélisation avancée des procédés thermiques rencontrés lors de la mise en forme des composites. $\mathrm{PhD}$ thesis, Ecole Centrale Nantes

12. Ghnatios C, Xu G, Leygue A, Visionneau M, Chinesta F, Cimetire A (2016) On the space separated represntation when addressing the solution of pde in complex domains. Discrete \& Continuous Dynamical Systems-Series S 9(2):475-500

13. Gruber MB, Lamontia MA, Tierney J, Gillespie JW (2009) Generating autoclave-level mechanical properties with out-ofautoclave thermoplastic placement of large composite aerospace structure. Technical report, National aeronautics and space administration, Langley research center

14. Hickey C, Timms JG, Bickerton S (2012) Compaction response and air permeability characterization of out-of-autoclave prepreg materials. In: Proceedings of the FPCM11 Confernece

15. Khan MA, Mitschang P, Schledjewski R (2010) Tracing void content development and identification of its effecting parameters during in situ consolidation of thermoplastic tape material. Polym Polym Compos 18(1):1-15

16. Leon A, Argerich C, Barasinski A, Soccard E, Chinesta F Effects of material and process parameters on in-situ consolidation. nternational Journal of Material Forming, In: Press

17. Leon A, Barasinski A, Chinesta F (2017) Microstructural analysis of pre-impreganted tapes consolidation. Int J Mater Form 10(3):369-378

18. Leon A, Barasinski A, Nadal E, Chinesta F (2015) High-resolution thermal analysis at thermoplastic pre-impregnated composite interfaces. Compos Interfaces 22(8):767-777

19. Leon A, Perez M, Barasinski A, Abisset-Chavanne E, Chinesta F (2017) On the properties evolution of engineered surfaces of thin reinforced thermoplastic tapes during consolidation. Surf Topogr Metrol Prop 5:044003

20. Perez M, Barasinski A, Courtemanche B, Ghnatios C, Chinesta F (2018) Sensitivity thermal analysis in the laser assisted tapeplacement process. Aims Materials Science 5(6):1053-1072

21. Pitchumani R, Ranganathan S, Don RC, Gillespie JW, Lamontia MA (1996) Analysis of transport phenomena governing interfacial bonding and void dynamics during thermoplastic tow-placement. Int J Heat Mass Transfer 39(9):1883-1897

22. Ranganathan S, Advani S (1995) A non-isothermal process model for consolidation and void reduction during in-situ tow placement of thermoplastic composites. J Compos Mater 29(8):10401062

23. Saoudi A, Leon A, Gregoire G, Barasinski A, Djebaili H, Chinesta F (2017) On the interfacial thermal properties of two rough surfaces in contact in preimpregnated composites consolidation. Surf Topogr Metrol Prop 5:045010

24. Simacek P, Advani S, Gruber M, Jensen B (2013) A nonlocal filling model to describe its dynamics during processing thermoplastic composites. Composites : Part A 46:154-165 
25. Stokes-Griffin CM, Matuszyk TI, Compston P, Cardew-Hall MJ (2012) Modeling automated tape placement of thermoplastic composites with in-situ consolidation. In: 4Th International Conference on Sustainable Automotive Technologies, vol 4. Springer, pp 61-68

26. Sequeira Tavares S, Caillet-Bois N, Michaud V, Manson JAE (2010) Non-autoclave processing of honeycomb sandwich structures Skin through thickness air permeability during cure. Composites : Part A 41:646-652
27. Tierney J, Gillespie JW (2003) Modeling of heat transfer and void growth for the thermoplastic composite tow-placement process. J Compos Mater 37:1745-1768

28. Zhang D, Levy A, Gillespie J (2012) On the void consolidation mechnism of continuous fiber reinforced thermoplastic composites. In: Sampe 2012, SAMPE, Baltimore 